\title{
Ship self-propulsion performance prediction by using OpenFOAM and different simplified propeller models
}

\author{
S. Gaggero, T. Gaggero, G. Tani, G. Vernengo, M. Viviani, D. Villa \\ University of Genoa, Department of Electrical, Electronic, Telecommunications Engineering and Naval \\ Architecture, Via Montallegro, 1, 16145 Genoa, Italy
}

\begin{abstract}
Classic hydrodynamics-related ship design problems can nowadays be approached by CFD viscous solvers. Ship self-propulsion performance prediction represents one of the most interesting problems in this framework. The capabilities of CFD codes to resolve accurately the separate problems (open water propeller performance and hull resistance) have been demonstrated over the last decades. The complexity of the combined problem (and, in turn, the required computational time) has restricted its solution to research applications still far from everyday industrial practice. Some approaches have been developed to reduce the computational burden, based e.g. on simple actuator-disk theory or, recently, on BEM/RANS coupled solvers. In this respect, different approaches exploiting the open-source solver OpenFOAM are presented, focusing on the main selfpropulsion parameters. In addition, a new numerical strategy able to provide more information compared to classical simplified approaches, is herein presented and validated against experimental measurements on the well-known Kriso Container Ship (KCS) test case.
\end{abstract}

\section{INTRODUCTION}

Over the last decades, Computation Fluid Dynamics (CFD) methods became a mature research tool to solve ship hydrodynamics problem and they also started to be considered for industrial applications at different stages of the design. This was possible thanks to the easier access to computational resources and to the theoretic improvements to the numerical solution of always more complex physical problems such as multi-phase and free surface flows. Considering these progress in hardware solutions and CFD techniques, some standard hydrodynamic problems in naval architecture can now be quite easily resolved in the framework of the so-called Virtual Towing Tank. This is the case for instance of performance prediction of the hull resistance advancing in calm water or of the propeller efficiency.

Both commercial and open-source software reach similar confidence levels when compared to experimental measurements (as shown in Larsson et al. 2015). All the applied solvers demonstrate to be able to predict the total hull drag in calm water within an error of $1-2 \%$ maximum. This is an encouraging result considering that the same accuracy has been shown in the experimental procedures reported by the International Toking Tank Conference (ITTC 2017), with different facilities performing the same test and cross-comparing each other measurements.
Recently, both modern commercial and opensource CFD software have been demonstrated to accurately predict e.g. both the planning hulls performance (Ferrando et al. 2015) as well as that of displacing ships (Gaggero et al. 2015). Moreover, it was possible to achieve these results at a relatively low computational cost. This is particularly important in order to allow to consider these type of simulations in early ship design stages and as viable alternative and complement (e.g. for typical medium size shipyards) to towing tank measurements. These modeling techniques, indeed, can also be used to support experimental campaigns, improving the knowledge of the flow field around the ship hull or for predicting the full-scale hull wake to the propeller to be further used as input for cavitation tunnel experiments (Tani et al. 2017) or final propeller design.

Accordingly, also propeller performance prediction by CFD methods has significantly grown (see, among the other, Gaggero et al. 2017a, Gaggero et al. 2010b, Gaggero et al. 2014b Gaggero et al. 2017d and Gaggero and Villa 2017b). The PPTC 2015 test case has been widely solved by using the open-source OpenFOAM libraries showing high accuracy both in cavitating and sub-cavitating regimes. Propeller design by optimization has been extensively and successfully completed by using both viscous unsteady Reynolds Averaged Navier Stokes (URANSE) solvers and ad-hoc developed Boundary Element Methods (BEM) (Gaggero et al. 2017c). 
Despite the aforementioned solutions are nowadays considered as everyday practice at least by large and medium-size companies, there are still several problems that are the object of research. This is mainly due to the relatively high computational effort required for those solutions, as the case of seakeeping (Grasso et al. 2010, Guo et al. 2012 and Deng et al. 2010, Bonfiglio et al. 2016) or ship manoeuvring in in calm water and waves (Sung and Park 2015, Ferrant et al. 2008).

Another complex problem is represented by selfpropulsion performance prediction, i.e. the evaluation of the propeller revolution rate when operating in the hull wake considering all the mutual interactions between the ship and the propeller (and possibly other appendages and/or the rudder). In addition, the equilibrium between the propeller thrust and the hull resistance at the design speed need to be reached. In fact, one of the main issues related to this condition is the increase in computational time. This is due to the very different time scales between the free surface flow around the hull and the flow closed to the propeller. Usually, the solver is constrained to impose a time-step defined by the faster flow dynamics, usually related to the propeller, while the total computational time is correlated to the hull flow, i.e. the one with the slower dynamics. The overall computational time can be much higher than those required to solve both problems separately. Simplified numerical strategies designed to reduce the overall computational effort, possibly limiting the loss of accuracy, have been recently proposed, ranging from simple actuator disk models (Carrica et al. 2013 and Fu et al. 2015) up to BEM/RANS coupled approaches (Villa et al. 2011, Gaggero et al. 2017c, Kim et al. 2006 and Alexander et al. 2009). Despite the propeller effects are included in the hull performance evaluation, the simplest methods based on actuator disks are not able to provide the complete set of data usually derived from self-propulsion tests (i.e. the propeller rate of revolution at equilibrium).

The proposed research focuses on the systematic comparison of different strategies, already successfully applied to investigate the rudder/propeller system (Bruzzone et al. 2014, Villa et al. 2017), to tackle the self-propulsion problem. In addition, a new procedure able to provide a wider set of useful information also from simplified actuator disk based calculations is presented and validated by comparison against available experimental measurements and higher fidelity (BEM/RANS coupling and full RANS analyses) calculations.

\section{TEST CASE}

The well-known KRISO Container Ship (KCS) is used as a numerical benchmark in the present study. Table 1 reports the main model data and Figure 1 shows a profile view of the underwater hull shape
(Larsson et al. 2015). A scale ratio of $1 / 31.6$ has been used. All the simulations have been performed at the ship design speed equal to 24 knots, corresponding to a Froude number of 0.26 . The self-propulsion prediction has been carried out considering the hull with its propeller but without the rudder to reproduce the same configuration adopted during the experimental measurements (as reported in Larsson et al. 2015). As requested by the ITTC' 78 procedure, a Skin Friction Correction (SFC) equal to $30.3 \mathrm{~N}$ is included. The towing tank test has been performed with the ship free to sink and trim. The dynamic attitude computed in towing conditions has been kept fixed during the selfpropulsion numerical simulations.

Figure 1. Sketch of the KCS test case hull shape.

Table 1 KCS main data in model scale

\begin{tabular}{lcc}
\hline Ship data in model scale & Symbol & Value \\
\hline Length between perp. & $\mathrm{L}_{\mathrm{PP}}[\mathrm{m}]$ & 7.2786 \\
Length of waterline & $\mathrm{L}_{\mathrm{WL}}[\mathrm{m}]$ & 7.3577 \\
Max. beam at waterline & $\mathrm{B}_{\mathrm{WL}}[\mathrm{m}]$ & 1.0190 \\
Draft & $\mathrm{T}[\mathrm{m}]$ & 0.3418 \\
Displacement volume & $\nabla\left[\mathrm{m}^{3}\right]$ & 1.6490 \\
Wetted surf. area w/o rud- & $\mathrm{S}_{\mathrm{W}}\left[\mathrm{m}^{2}\right]$ & 9.4379 \\
der & & \\
Propeller center, (from FP) & $\mathrm{x} / \mathrm{L}_{\mathrm{PP}}$ & 0.9825 \\
Propeller center, (below & $\mathrm{z} / \mathrm{L}_{\mathrm{PP}}$ & -0.02913 \\
WL) & & \\
Prop. rotation dir. & & clockwise \\
\hline
\end{tabular}

\section{NUMERICAL MODEL: APPROXIMATION OF THE PROPELLER EFFECT}

The open source OpenFOAM libraries have been used to investigate the KCS self-propulsion condition. This suite provides a wide type of solvers and pre/post processors designed to solve most of the flow problem in the engineering field. One of the goals of this work is, in fact, to test a full license-free approach on a particular and complex naval hydrodynamic problem. In this light, both the built-in Cartesian hexdominant mesher snappyHexMesh and the interDyMFoam solver have been applied. The necessary tuning of this meshing tool often represents the bottleneck of such kind of applications but its rich features, which can be freely customized and parameterized, ensure the generation of high-quality meshes, mandatory for the so-called "Virtual toking tank" computations.

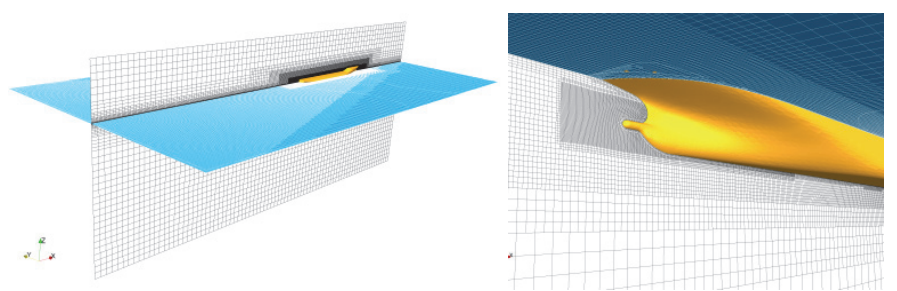

Figure 2. KCS mesh layout with hull wake detail (right). 
Figure 2 shows an overview and a close-up of the generated mesh. The figure highlights the free-surface vertical refinement, essential to properly capture the hull generated wave pattern, and the horizontal refinements inside the Kelvin region (which extends for about $\pm 20^{\circ}$ from the bow of the hull). In addition, due to the need of resolving the hull-propeller interaction, a finer mesh has been clustered on this region. A final mesh of about 3 million cells is used for the whole computational domain.

Regarding the solver set-up, two approaches have been used to address time discretization: the Local Time Step (LTS) approach for steady solutions and an implicit time marching scheme for transient solutions. The two approaches need different computational resources: the first is faster but can be only used if a steady free surface solution is expected while the latter, that is the slower, requires higher computational resources because the solution time-step is constrained by the maximum admissible Courant number. Turbulence has been modeled using the SST $k-\omega$ formulation. A more detailed description of the mesh, domain and of solvers characteristics can be found in Gaggero et al. (2015) and Gaggero et al. (2017a).

As mentioned, the exploration of the cost/benefit balance resulting from different approaches when including the effects of the propeller working behind the hull is one of the main goals of this research. Several approaches have been considered with different levels of complexity:

- The simpler constant translational actuator disk which includes only the thrust force $\left(C D_{T}\right)$, uniformly distributed over the entire propeller disk (Figure 3a),

- The rotor-translational disk, which includes also the torque moment $\left(C D_{T Q}\right)$, uniformly distributed over the entire disk (Figure 3b),

- The radially varying actuator disk, which considers the radial distribution of propeller force and moment $\left(R D_{T Q}\right)$, as per Figure $3 \mathrm{c}$,

- The radial-tangential varying actuator disk which considers also the circumferential variation of forces generated by propeller in a non-uniform wake $\left(R T D_{T Q}\right)$, as per Figure $3 \mathrm{~d}$,

- The BEM/RANS coupled approach (Figure 3e), originally developed in Villa et al 2011.

- The full-RANS approach (Figure 3f) where the propeller is fully resolved in the RANS solver. This is used as the reference.

The comparison of the costs/benefits of different propeller approximations has been previously analyzed in Bruzzone et al. (2014). Those methods have been used to resolve the propeller/rudder interaction, comparing the generated flow fields and focusing on the accelerated flow field and its interaction with the rudder. In the present paper, due to the absence of the rudder, the focus will be on the model interaction with a body (the hull) located in front of the propeller region.

A dedicated implementation of these actuator disks in the OpenFOAM framework has been developed adding a new fvOption class method. Each of the proposed models requires a different level of knowledge of the propeller geometry and functioning. This makes them suitable at different ship design stages. For example, the BEM/RANS coupled approach requires the detailed propeller geometry knowledge and the availability of an unsteady BEM solver, restricting its application only at an advanced design stage. On the contrary, the $C D_{T}$ model requires only the propeller diameter, commonly known since the preliminary design stages.

Figure 3 displays some perspective views of the described models with their own forces distributions.

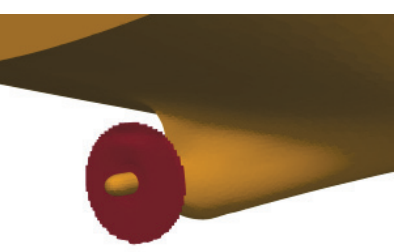

(a) Constant Disk - Thrust

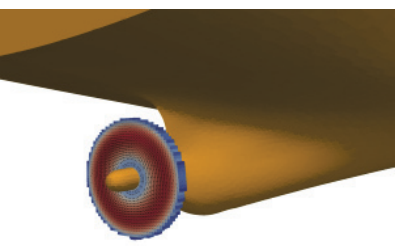

(c) Radial distributions of Thrust and Torque

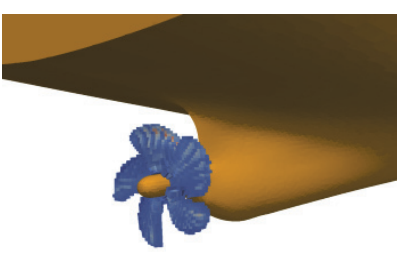

(e) Coupled BEM/RANS

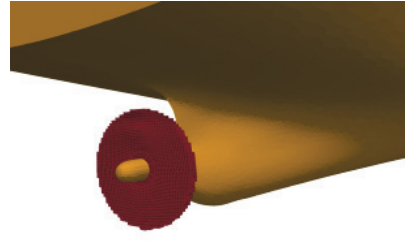

(b) Constant Disk - Thrust and Torque

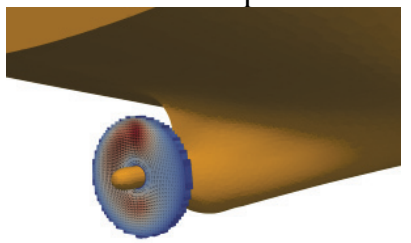

(d) Radial/tangential distributions of Thrust and Torque

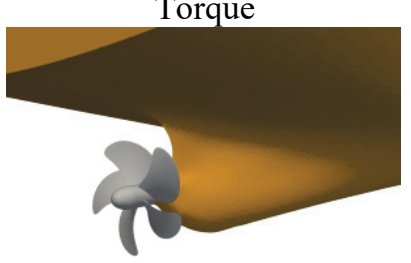

(f) full RANS
Figure 3. Distribution of forces imposed in the simplified models and propeller geometry.

The two simpler models, $C D_{T}$ and $C D_{T Q}$, distribute uniformly the thrust force on the whole propeller disk region; the latter adds also a homogeneous tangential force proportional to the imposed torque. These models require only the knowledge of the propeller diameter and, for the second one, a preliminary estimation of the propeller efficiency, being the thrust always equal to the ship drag. These data commonly are known or can be estimated, since from the preliminary ship design stages. As demonstrated in Bruzzone et al. (2014), these simple and efficient models are not able to generate a reliable propeller wake, which can be obtained instead by the radial varying actuator disk model. The $R D_{T Q}$ approach distributes both axial and 
tangential forces following expected blade force distributions along the radius (those computed from a lifting line design approach, for instance), therefore increasing the axial force at $\mathrm{r} / \mathrm{R}$ about equal to 0.7 . This model, which has been previously demonstrated to be able to reasonably predict the propeller wake field, is not able to take into account the blade force variation during a revolution due to the presence of the non-uniform hull wake. The influence of this unsteadiness is, instead, partially included with the $R T D_{T Q}$ model, which shows, as in Figure 3, the typically increased distribution of forces on the upper-left part of the disk where a slower wake interacts with the propeller blade, increasing its load. Both $R D_{T Q}$ and $R T D_{T Q}$ models, compared to the constant disk ones, require more detailed propeller information (propeller force radial and/or tangential distributions and unsteady functioning), which can be provided, for example, by a simplified potential method as lifting line/lifting surface, BEMT or BEM codes. In the present work, these data have been computed by a dedicated BEM code (Gaggero 2010a), which has been also used to compute the body force distributions necessary for the BEM/RANS coupling. Behind the BEM/RANS coupling, indeed, there is the prediction of unsteady blade forces with the Panel Method, which turn into the unsteady body forces to be included into the RANS domain, and the iterative prediction of the effective wake by subtracting the propeller self-induced velocities (BEM) from the total velocity field (RANS) in front of the propeller.

\subsection{Self-propulsion procedure}

One of the most challenging aspects related to the self-propulsion simulation is the evaluation of the self-propulsion parameters (thrust deduction factor and wake factor) especially when simplified models, like actuator disks, are used.

When a full-RANS approach is used, the same procedure adopted in the experimental activities can be implemented. Setting the desired ship velocity, an initial propeller revolution rate is imposed (commonly defined by means of the nominal wake and the open water propeller curves), then, computing both the hull actual drag and the provided propeller thrust, the revolution rate is varied until an equilibrium condition is obtained (considering also the SFC factor). As a result, the equilibrium thrust (T), the resulting thrust deduction factor $(\mathrm{t})$, the torque $(\mathrm{Q})$ and propeller RPS are found and, by means of the propeller open water curves, as prescribed by ITTC' 78 procedures, the effective wake fraction (w) can be computed.

When any actuator disk model is used, differently, only the equilibrium thrust and the consequently thrust deduction factor can be directly determined by the simulation. The effective wake fraction, on the contrary, needs a dedicated analysis. Considering that the propeller open water curves cannot be used because the propeller rate of rotation is unknown, the wake fraction can be directly evaluated by the integral of the velocity field in the propeller plane, as preliminarily shown in Villa et al. (2011). Unlikely, this data, due to the presence of the body force region, is affected also by the self-induced velocities generated by the momentum source represented by the actuator disk. The proposed procedure intends to evaluate this latest contribution by means of a dedicated open water simulation where the same actuator disk used for self-propulsion operates in a homogeneous mean nominal wake. In such way, an approximated self-induced velocity field in the propeller plane (inside the body force disturbed region) can be directly evaluated and by subtracting it (Equation 1) from the total velocity field, the effective wake can be determined:

$$
\begin{gathered}
V_{\text {effective }}=V_{\text {total }}-\left(\begin{array}{c}
V_{\text {actuator disk }}^{\text {in nominal wake }}-V_{\text {ship }} \\
\left.\cdot(1-w)_{\text {nominal }}\right)
\end{array}\right)
\end{gathered}
$$

where $V_{\text {total }}$ is the "total" velocity (Carlton, 2007) field computed by RANS on the propeller plane with the influence of the actuator disk and $V_{\text {actuator disk in nominal wake }}$ is the "total" velocity field on the propeller plane (including the influence of the actuator disk) of a simplified analysis where the actuator disk, operating in open water condition, is subjected to an uniform inflow equivalent to the nominal wake of the ship in towing condition $\left(V_{\text {ship }}\right.$. $\left.(1-w)_{\text {nominal }}\right)$.

Once the effective wake (by Equation 1) and the thrust deduction factor are known, also the propeller revolution rate can be estimated by means of the numerical (or experimental if available) open water propeller curves.

This procedure for the calculation of the effective wake, at the cost of a simple and trivial additional calculation (actuator disk in uniform inflow equivalent to nominal wake), has been adopted for all the actuator disk models. Differently, in the BEM/RANS coupled approach, the effective wake has been estimated by directly computing the unsteady self-induced velocities in front of the propeller using the Panel Method and iteratively exchanging body forces at a given revolution rate (from BEM to RANS) and total velocity fields (from RANS to BEM) as described in Gaggero et al. (2017c). The computed effective wake in one or more plane in front of the propeller are then extrapolated to the propeller plane, to have a more reliable estimation of the effective wake. The functioning conditions (thrust and the relative propeller rate of revolution) are consequently directly provided by the unsteady BEM calculations.

An example of the application of Equation 1 is presented in Figure 4, where the mean wake fractions on a region equivalent to the propeller disk are computed at different longitudinal positions, being $\mathrm{x} / \mathrm{D}=0$ the 
propeller plane (negative forward). In particular, the comparison is among the nominal wake (ship in towing condition), the "total" wake in self-propulsion condition (RANS with the $C D_{T}$ actuator disk model) and the "total" wake of the same actuator disk model subjected to a uniform open water inflow equivalent to the nominal wake of towing condition $\left(V_{\text {ship }}\right.$. $\left.(1-w)_{\text {nominal }}\right)$. The resulting effective wake does not show anymore the "jump" due to the momentum sources representing the action of the propeller across $\mathrm{x} / \mathrm{D}=0$ and, as expected, it is possible to appreciate that it is "faster" than the nominal wake while maintaining a very similar behavior.

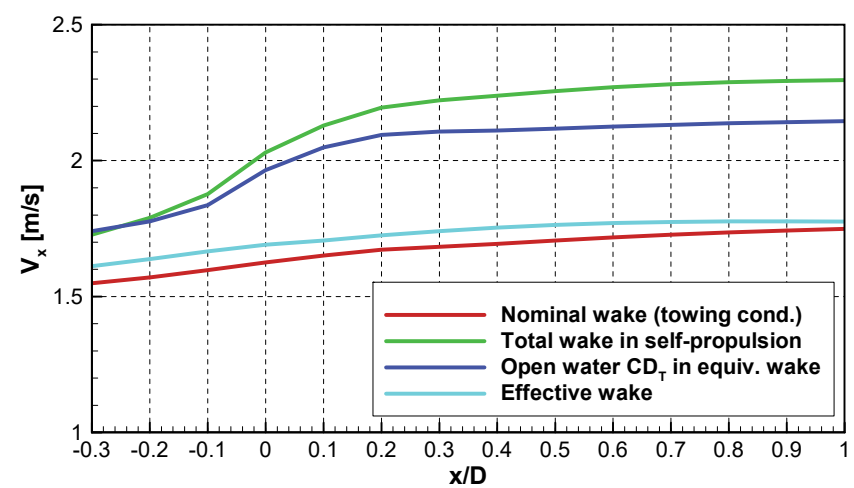

Figure 4. Example (model scale with $\mathrm{CD}_{\mathrm{T}}$ ) of the wake fraction variation along the longitudinal direction.

This analysis, which has been applied to the wake fraction seen as the average of the velocities on the propeller plane, can be identically applied point by point on the propeller plane, opening (as shown in the next section) the way to the adoption of this procedure also for non-equilibrium conditions, as maneuvering ones.

\section{RESULTS}

Preliminarily to self-propulsion analyses, towing tank tests have been carried out and compared with literature data. Results are summarized in Table 2. A discrepancy lower than $1 \%$ has been found for the total drag coefficient and even lower discrepancies are present for the ship attitude. Results of present analyses, which are within the numerical accuracy reported in the Tokyo 2015 Workshop where several solvers and users experience have been compared, can be considered acceptable. To have a complete insight into the reliability of the simplified procedure based on actuator disk calculations proposed to estimate the self-propulsion propeller/hull interactions, it is important to verify also the accuracy in predicting the nominal wake, both in terms of average and local values. Results of this analysis are summarized in Table 2 and in Figure 5.

Differences in terms of average values are less than $3 \%$. The computed nominal wake is slightly faster than the measured wake and this is mainly due to the underestimation of the velocity reduction in correspondence of the hull stern while maintaining an overall good similarity in terms of local distribution of velocity.

Table 2. CFD and EFD comparison of the KCS towing tank test with model free to sink and trim.

\begin{tabular}{lccccc}
\hline KCS ship & Sinkage & Trim & $\begin{array}{c}\mathrm{C}_{\mathrm{T}} \\
\cdot 10^{3}\end{array}$ & $\mathrm{C}_{\mathrm{F}} \cdot 10^{3}$ & $1-\mathrm{W}$ \\
\cline { 2 - 3 } & {$[\mathrm{cm}]$} & {$[\mathrm{deg}]$} & & & \\
\hline CFD & 1.391 & -0.170 & 3.674 & 2.897 & 0.739 \\
EFD & 1.395 & -0.169 & 3.711 & 2.883 & 0.719 \\
\hline
\end{tabular}
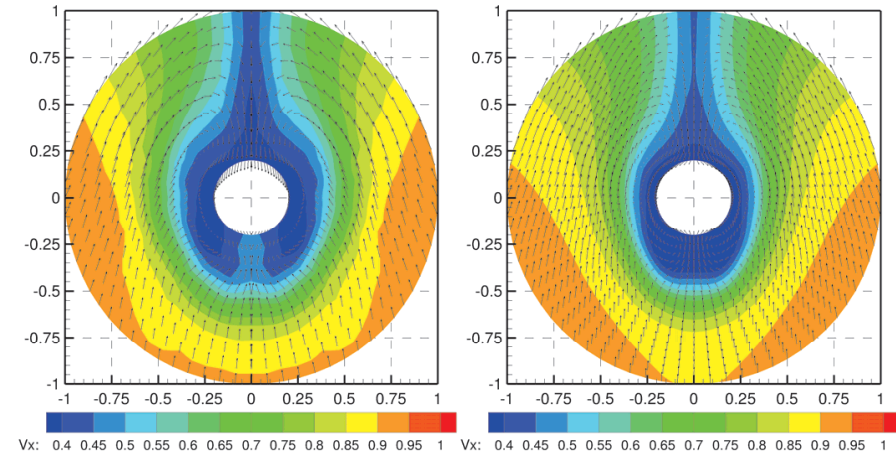

Figure 5. Comparison between EFD (left) and CFD (right) nominal wakes for the KCS in model scale at 0.26 Froude number

Self-propulsion simulations have been carried out using all the propeller approaches outlined in Section 3. When simplified propeller methods $\left(C D_{T}\right.$ to BEM/RANS coupling) have been adopted, calculations have been carried out exactly with the same mesh of the towing test analyses. On the contrary, for full RANS analyses, sliding meshes have been employed by using a dedicated inner, rotating, region where the propeller has been modelled. The exact representation of the propeller required 1 Million of additional cells, which significantly contributes to the total cells count.

Table 3 Self-propulsion parameters comparison varying the actuator disk with the experimental data as reference

\begin{tabular}{lcccc}
\hline \multirow{2}{*}{ Model } & Prop. Rev. & Thrust & $1-\mathrm{w}$ & $1-\mathrm{t}$ \\
\cline { 2 - 3 } & {$[\mathrm{RPS}]$} & {$[\mathrm{N}]$} & & \\
\hline $\mathrm{CD}_{\mathrm{T}}$ & 9.533 & 61.50 & 0.7695 & 0.8871 \\
$\mathrm{CD}_{\mathrm{TQ}}$ & 9.581 & 62.50 & 0.7713 & 0.8710 \\
$\mathrm{RD}_{\mathrm{TQ}}$ & 9.581 & 62.66 & 0.7704 & 0.8701 \\
$\mathrm{RTD}_{\mathrm{TQ}}$ & 9.637 & 62.89 & 0.7778 & 0.8667 \\
$\mathrm{BEM} /$ RANS & 9.570 & 64.20 & 0.7816 & 0.8494 \\
Full-RANS & 9.656 & 62.77 & 0.7618 & 0.8688 \\
Exp. & 9.500 & 65.15 & 0.7860 & 0.8460 \\
\hline
\end{tabular}

Furthermore, when the BEM/RANS coupling or the full-RANS approach is used, an unsteady solver is necessary. This aspect further contributes to the computational weighting of the calculations with respect to the actuator disk models that, by representing the propeller as its average action on the flow, operate in steady mode. The increase of the computational time is particularly significant for the full-RANS analyses, being the simulation time step (about $0.5^{\circ}$ of propeller rotation) strictly constrained by sufficiently low Courant numbers. Table 3 reports a summary of the computed results for each propeller 
model compared to the experimental measures. The first four models $\left(C D_{T}\right.$ to $\left.R T D_{T Q}\right)$ use Equation 1 for the estimation of the effective wake and, coherently with their application (radial and tangential distribution of forces are obtained from initial estimation of propeller performances using BEM), make use of the open water propeller curves from BEM calculations to estimate the propeller rate of revolution. For the BEM/RANS coupling, the propeller rate of revolution is a direct result of the analysis and the wake fraction is the spatial average of the distribution of velocities on the propeller disk once propeller self-induction is subtracted, at convergence, from total velocities. For full RANS, instead, the wake fraction is obtained, in analogy to ITTC'78 experimental procedures, from the open water propeller curves estimated with the RANS itself.

According to the reported results, it is evident that the interaction between the hull and the simplified propeller models is only slightly influenced by the adopted approach. The thrust at equilibrium (and, in turn, the thrust deduction factor) varies less than $4 \%$, with a discrepancy in terms of predicted propeller rate of revolution lower than $1 \%$. In addition, a certain convergence trend can be appreciated by looking at the results provided by the actuator disks models. As the model increases its complexity (from constant to radial and to radial/tangential distribution of forces), the predicted rate of revolution tends to the most complete, full RANS, computation (even if discrepancies between open water propeller curves by BEM and RANS should be taken into account). BEM/RANS coupling, instead, shows slightly different results which, in the light of the analyses carried in Gaggero et al. (2014a), can be ascribed to the differences between equivalent open water calculations and unsteady analyses with BEM when significantly radially varying wakes are considered. Overall, the agreement with experiments is good, also in the light of the numerical results submitted to the Tokyo 2015 Workshop (Larsson et al. 2015). In this Workshop, the average predicted propeller rate of revolution was equal to 9.576 with a quite large dispersion of the data $( \pm 0.2$ RPS): current results, also those obtained with the simplified models, are exactly within the confidence reported in the Workshop.

Figure 6 reports the velocity flow field computed in the propeller plane, then subjected to the acceleration provided by the actuator disk models. Several aspects can be highlighted.

The $C D_{T Q}$ model, compared to the $C D_{T}$ one, shows the rotational component of the velocity field related to the inclusion of the tangential momentum sources proportional to the torque.

The distribution of axial velocity is not significantly affected by the inclusion of tangential forces and the negligible differences at outer radii can be

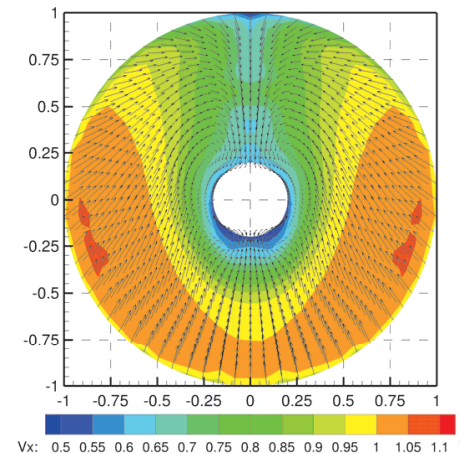

(a) $\mathrm{CD}_{\mathrm{T}}$ model

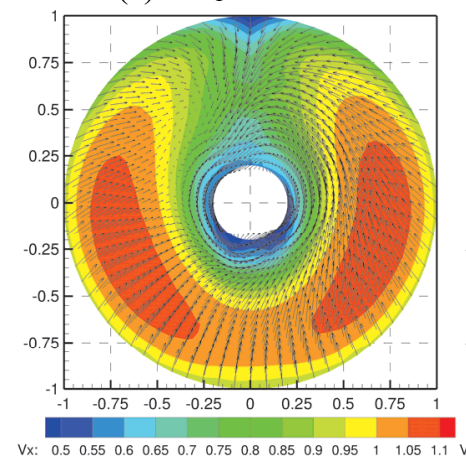

(c) $\mathrm{RD}_{\mathrm{TQ}}$ model

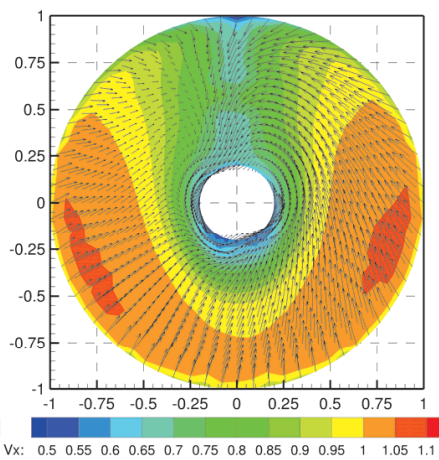

(b) $\mathrm{CD}_{\mathrm{TQ}}$ model

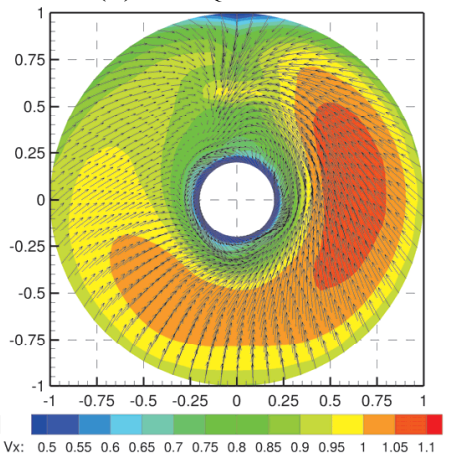

(d) RTD $_{\mathrm{TQ}}$ model
Figure 6. Computed wake in self-propulsion mode in the propeller plane (seen from aft). (a) $C D_{T}$, (b) $C D_{T Q}$, (c) $R D_{T Q}$ and (d) $R T D_{T Q}$

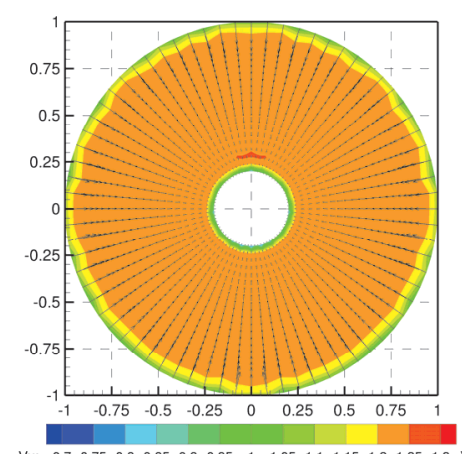

(a) $\mathrm{CD}_{\mathrm{T}}$ model

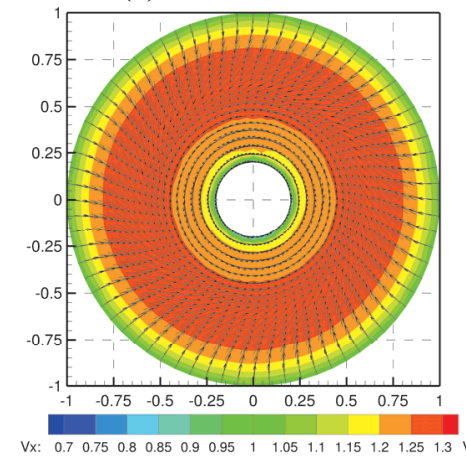

(c) $\mathrm{RD}_{\mathrm{TQ}}$ model

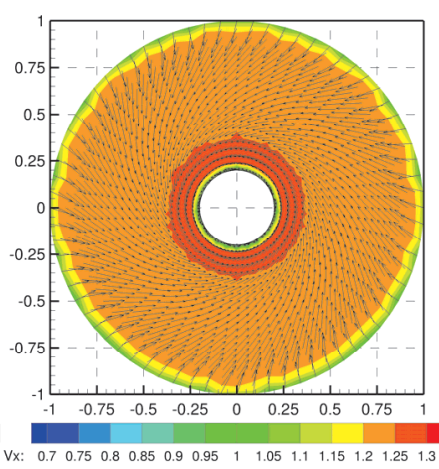

(b) $\mathrm{CD}_{\mathrm{TQ}}$ model

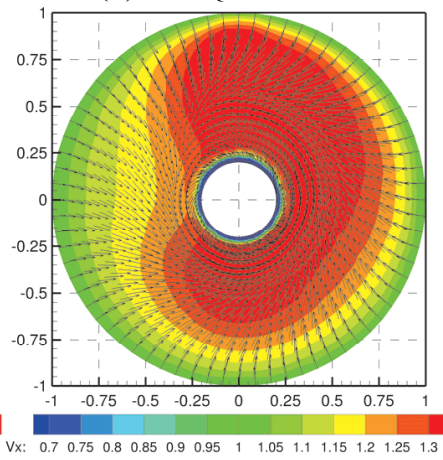

(d) $\mathrm{RTD}_{\mathrm{TQ}}$ model
Figure 7. Induced velocity fraction during open water test in the propeller plane. (a) $C D_{T}$, (b) $C D_{T Q}$, (c) $R D_{T Q}$ and (d) $R T D_{T Q}$.

ascribed mainly to the slightly different equilibrium point (i.e. higher momentum sources) predicted by the actuator disk accounting also for torque.

Differently, the $R D_{T Q}$ model shows, as expected, an increased axial velocity in correspondence of $\mathrm{r} / \mathrm{R}=0.7$, where the propeller load, and in turn, the 

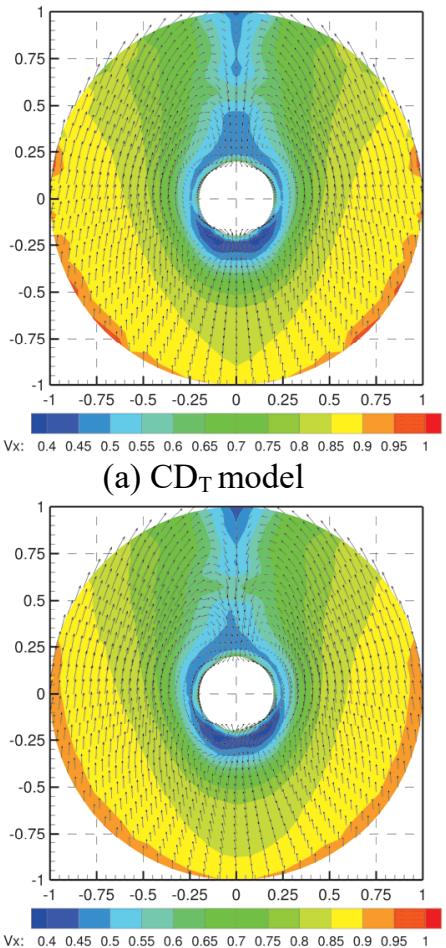

(c) $\mathrm{RD}_{\mathrm{TQ}}$ model (a) $\mathrm{CD}_{\mathrm{T}}$ model

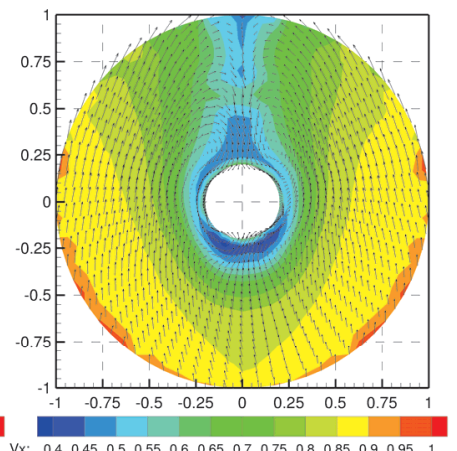

(b) $\mathrm{CD}_{\mathrm{TQ}}$ model

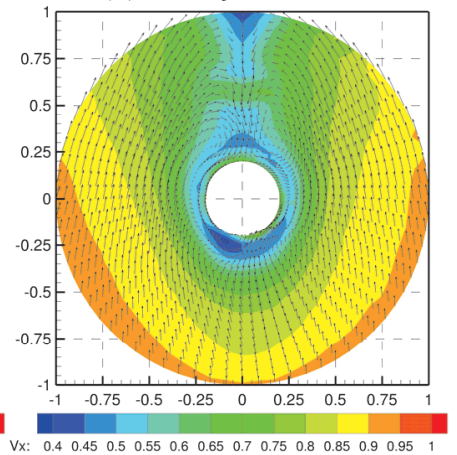

(d) RTD TQ model

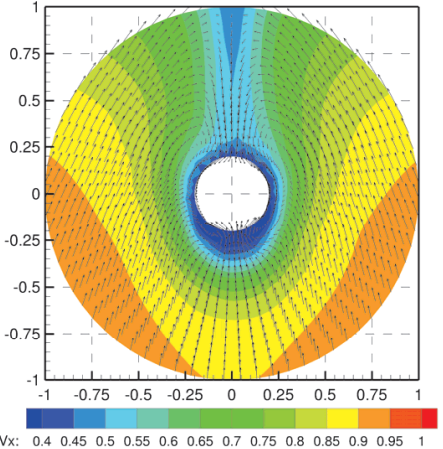

(e) BEM/RANS coupled model

Figure 8. Predicted effective wake for the simplified propeller models. (a) $C D_{T}$, (b) $C D_{T Q}$, (c) $R D_{T Q}$, (d) $R T D_{T Q}$ and (e) BEM/RANS coupled model.

momentum sources, computed by the BEM, is higher. All these models $\left(C D_{T}, C D_{T Q}\right.$, and $\left.R D_{T Q}\right)$, as previously mentioned, are axially symmetrical, therefore do not include the different load experienced by the blades during a single revolution. The $R T D T Q$ model, on the contrary, adds this effect by including the actual distribution of momentum sources at any angular position based on unsteady calculations by the Panel Method with the preliminary nominal wake as the spatial non-homogeneous inflow. This peculiarity is well observable in Figure 6d where the higher axial velocities on the starboard side are representative of the higher blade load when it operates into the tangential flow of Figure 5. These differences (and peculiarities) among the various actuator disk models are furthermore evidenced in Figure 7, where the propeller induced velocities are extracted from the equivalent open water tests needed by Equation 1. Almost uniform distribution of axial self-induced velocities when constant actuator disks $\left(C D_{T}\right.$ and $\left.C D T Q\right)$ are used, axisymmetric radial and tangential components when torque is added to the models $\left(C D_{T Q}\right)$, higher self-induced velocities in correspondence of higher local loads $(R D T Q$ and $R T D T Q$ respectively for radial and radial/circumferential distributions of body forces) can be highlighted as well.

Figure 8, finally, shows the comparison of the effective wakes obtained by applying Equation 1 with the four proposed actuator disk models and with the extrapolated effective wake on the propeller plane from the BEM/RANS coupling. Regardless the actuator disk model, predictions are very similar, both in terms of wake fractions (Table 3) and local distribution of velocities, to the effective wake by the BEM/RANS coupling which, among the approaches for the prediction of the effective wake as the difference between the total and the self-induced velocity fields (Carlton 2017), can be considered the most accurate implementation. This confirms the consistency and the reliability of the simplified approach, which results consequently useful also for an initial estimation of the flow field for wake adapted propeller design.

\section{CONCLUSION}

A systematic analysis of different methods to include the propeller effect in a viscous RANS solver to simulate a ship in self-propulsion condition has been carried out. Four simplified actuator disks models have been explored, comparing the results, both in terms of global self-propulsion parameters and local flow field variables, with the BEM/RANS coupling approach, with full RANS calculations, and with the available experimental measurements.

Results from any of the proposed methods are good, especially in the light of the computational efficiency provided by the simplified approaches, which, consequently, could be applied during the preliminary design phases with an already satisfactory level of confidence. The propeller rate of revolution is overestimated by all the methods but the highest difference (with the full RANS approach) is, however, lower than $1.5 \%$. In this particular case, a certain underprediction (also in open water condition) of the propeller forces at moderate/high advance coefficients by RANS, widely evidenced in literature, could explain the highest predicted propeller rate of revolution.

For the thrust deduction factor and the wake fraction, the differences between measurements and predictions with the simplified actuator disk models or with the BEM/RANS coupling are almost negligible. The highest differences (when the simplest model, $C D_{T}$, is applied), for both the quantities, are respectively of $5 \%$ and $2 \%$. As the complexity of the model increases, discrepancies are between 1 and $2.5 \%$, which can be considered more than satisfactory in the light of simplification introduced in the model. As in the case of the prediction of the propeller rate of revolution, full RANS calculations, especially for the 
prediction of the wake fraction, are significantly influenced by the inaccurate prediction of the open water propeller curves.

Also locally, the simplified approaches predict, almost regardless the considered model, very similar distributions of the effective velocity field. Together with the computational efficiency of the models (even higher than that of the BEM/RANS coupling which is usually considered a standard for an estimation of the effective wake field) this is a key point of the proposed analysis, opening the opportunity to have, in a very preliminary design phase, a reliable estimation of all the quantities necessary for a wake adapted propeller design.

The required steps for an even more robust approach consist of an extensive validation of these simplified models, including in the analyses configurations with shaft brackets, rudders and severe hull sterns generating strongly disturbed wakes.

\section{REFERENCES}

Alexander, B., Phillips, S., Turnock, R. and Furlong, M., 2009: Evaluation of manoeuvring coefficients of a self-propelled ship using a blade element momentum propeller model coupled to a Reynolds averaged Navier Stokes flow solver, Ocean Engineering, Volume 36, Issues 15-16, 2009, pp. 1217-1225.

Bonfiglio, L., Vernengo, G., Brizzolara, S. and Bruzzone, D. 2016: A hybrid RANSE - strip theory method for prediction of ship motions. Proceedings of the $3^{\text {rd }}$ International Conference on Maritime Technology and Engineering, MARTECH 2016, in: Marine Technology and Engineering, 3, CRC Press, Editors: Guedes Soares \& Santos, Volume 1, pp. 241250.

Bruzzone, D., Gaggero, S., Podenzana Bonvino, C., Villa, D. and Viviani, M., 2014: Rudder-Propeller Interaction: analysis of different approximation techniques, Proceedings of the 11th International Conference on Hydrodynamics ICHD 2014, Singapore, October 19-24 2014, pp. 230-239.

Carlton, J.S., (2007) Marine Propellers and Propulsion, 2nd edition, Butterworth-Heinemann, 2007.

Carrica, P.M., Ismail, F., Hyman, M., Bhushan, S. and Stern F. 2013: Turn and zigzag maneuvers of a surface combatant using a URANS approach with dynamic overset grids. J Mar Sci Technol 18:166.

Deng, G.B., Queutey, P. and Visonneau, M., 2010: RANS prediction of the KVLCC2 tanker in head waves, Journal of Hydrodynamics, Ser. B, Volume 22, Issue 5, Supplement 1, 2010, pp. 476-481.

Ferrando, M., Gaggero, S. and Villa, D. 2015: Open Source Computational of Planing Hull Resistance, Transactions of the Royal Institution of Naval Architects Part B: International Journal of Small Craft Technology, Vol 157, Issue JulDec 2015, pp. 83.98, , Royal Institution of Naval Architects.

Ferrant, P., Gentaz, L., Monroy, C., Luquet, R., Ducrozet, G., Alessandrini, B., Jacquin, E. and Drouet, A., 2008: Recent advances towards the viscous flow simulation of ships manoeuvring in waves, Proceedingd of 23rd International Workshop on Water Waves and Floating Bodies, Jeju, Korea.

Fu, H., Michael, T. J. and Carrica, P. M., 2015: A method to perform self-propulsion computations with a simplified body-force propeller model, The Twenty-fifth International
Ocean and Polar Engineering Conference, International Society of Offshore and Polar Engineers.

Gaggero S., 2010a: Development of a potential panel method for the analysis of propellers performances in cavitating and supercavitating conditions, Ph.D. Thesis, University of Genoa, Italy, 2010 (in Italian).

Gaggero, S., Villa, D. and Brizzolara, S., 2010b: RANS and PANEL method for unsteady flow propeller analysis, Journal of Hydrodynamics, 22 (5 SUPPL. 1), pp. 547-552.

Gaggero, S., Villa, D. and Viviani, M., 2014a: An investigation on the discrepancies between RANS and BEM approaches for the prediction of marine propeller unsteady performances in strongly non-homogeneous wakes, Proceedings of the 33rd International Conference on Ocean, Offshore and Artic Engineering, OMAE 2014, San Francisco, USA, June 201, pp 1-13

Gaggero, S., Villa, D., Viviani, M. and Rizzuto, E., 2014b: Ship wake scaling and effect on propeller performances. Proceedings of IMAM 2013, 15th International Congress of the International Maritime Association of the Mediterranean, in: Developments in Maritime Transportation and Exploitation of Sea Resources, CRC Press, Editors: Guedes Soares \& Lopez-Pena, Volume 1, pp. 13-21.

Gaggero, S., Villa, D. and Viviani, M., 2015: The Kriso Container Ship (KCS) test case: an open source overview, Proceedings of VI International Conference on Computational Methods in Marine Engineering, MARINE 2015, June 15 17, Rome, Italy pp. 735-749.

Gaggero, S., Tani, G., Villa, D., Viviani, M., Ausonio, P., Travi, P., Bizzarri, G. and Serra, F., 2017a: Efficient and multi-objective cavitating propeller optimization: An application to a high-speed craft, Applied Ocean Research, Volume 64, pp. 31-57.

Gaggero, S. and Villa, D., 2017b: Steady cavitating propeller performance by using OpenFOAM, StarCCM+ and a boundary element method, Proceedings of the Institution of $\mathrm{Me}$ chanical Engineers Part M: Journal of Engineering for the Maritime Environment, 231 (2), pp. 411-440.

Gaggero, S., Villa, D. and Viviani, M., 2017c: An extensive analysis of numerical ship self-propulsion prediction via a coupled BEM/RANS approach, Applied Ocean Research, Volume 66, pp. 55-78.

Gaggero, S. and Villa, D., 2017d: Cavitating Propeller Performance in Inclined-Shaft Conditions with OpenFOAM: the PPTC 2015 test case, Accepted for publication on Journal of Marine Science and Applications (JMSA-2016-09-0083)

Grasso, A., Villa, D., Brizzolara, S. and Bruzzone, D., 2010: Nonlinear motions in head waves with a RANS and a potential code, Journal of Hydrodynamics, Volume 22, Issue 5, Supplement 1, pp. $10-15$.

Guo, B.J., Steen, S. and Deng, G.B., 2012: Seakeeping prediction of KVLCC2 in head waves with RANS, Applied Ocean Research, Volume 35, 2012, Pages 56-67.

Kim, J., Kim, K.S., Kim, G.D., Park, I.R. and Van S.H., 2006: Hybrid RANS and potential based numerical simulation for self-propulsion performances of the practical container ship J Ship Ocean Technol, 10 (4), pp. 1-11.

Larsson, L., Stern, F., Visonneau, M., Hirata, N., Hino, T., and Kim, J., 2015: Tokyo 2015: A workshop on CFD in ship hydrodynamics. In Proceedings (Vol. 2).

Resistance Committee, 2017: Final Report and Recommendations to the 28th ITTC, Proceedings of 28th International Towing Tank Conference, Vol 1, Wuxi, China.

Sung, Y. J., and Park, S. H., 2015: Prediction of ship manoeuvring performance based on virtual captive model tests, 
Journal of the Society of Naval Architects of Korea, 52(5), 407-417.

Tani, G., Viviani, M., Villa, D. and Ferrando, M., 2017: A study on the influence of hull wake on model scale cavitation and noise tests for a fast twin-screw vessel with inclined shaft, Proceedings of the Institution of Mechanical Engineers Part M: Journal of Engineering for the Maritime Environment, pp. 1-24.
Villa, D., Gaggero S. and Brizzolara, S., 2011: Simulation of ship in self propulsion with different CFD methods: from actuator disk to potential flow/RANS coupled solvers, Proceedings of International Conference-Developments in Marine CFD RINACFD2011, London, England, 22-23 March 2011.

Villa, D., Viviani, M., Tani, G., Gaggero, S., Bruzzone, D. and Podenzana Bonvino, C., 2017: Numerical Evaluation of Rudder Performance Behind a Propeller in Bollard Pull Condition, Accepted for publication on Journal of Marine Science and Applications. 